First publ. in: Ultramicroscopy 71 (1998), 1-4, pp. 345-350

\title{
An approach to spectroscopy and phase measurements in scanning plasmon near-field microscopy
}

\author{
J. Boneberg*, M. Ochmann, H.-J. Münzer, P. Leiderer \\ Universität Konstanz. Postfach 5560 M676, D-78434 Konstanz. Germany
}

\begin{abstract}
A new optical setup for plasmon near-field optical microscopes is introduced which allows to improve the signal/background ratio independently of the wavelength used and therefore makes the spectroscopy feasible. Calculated and experimental examples demonstrate this improvement obtained by addition of ellipsometric components. Furthermore, a first measurement in combination with a scanning tunnelling microscope shows that it is possible to extrude phase information from this setup as well.
\end{abstract}

PACS: $07.60 . \mathrm{Pb} ; 07.60 . \mathrm{F}$

Keywords: NFOM; Spectroscopy in NFOM

\section{Introduction}

Different setups of apertureless near-field microscopes $[1-7]$ have been shown to achieve a high lateral resolution compared to other types of nearfield microscopes as the Near-Field Scanning Optical Microscope (NSOM). For example, the Scanning Plasmon Near-Field Microscope (SPNM) which, in principle, is a combination of surface-plasmon excitation in Attenuated Total Re-

*Corresponding author. Tel: +497531 883562; fax: + 49 7531 883127; e-mail: johannes.boneberg(a)uni-konstanz.de. flection geometry (ATR) with a Scanning Tunnelling Microscope (STM) has already been introduced several years ago [3]. In these measurements, a lateral resolution of $\lambda / 200$ was shown. A similar setup in combination with an Atomic Force Microscope (AFM) achieved a resolution of about $20 \mathrm{~nm}$ [5]. Beside the high lateral resolution the ATR setup has the advantage of an additional field enhancement at the surface [8]. Nevertheless, almost no applications of these methods have been shown. One restriction is the poor signal-to-background ratio. Even as this ratio could be improved for one single wavelength by proper choice of the metalfilm thickness, spectroscopic measurements have not been possible caused by a rapidly increasing 
background signal for other wavelengths. The availability of spectroscopic data would also relieve the interpretation of the optical contrast which is another main problem for the lack of application of these methods.

In this paper we show that the addition of an ellipsometric like setup to the ATR-configuration allows to improve the signal-to-background ratio independently of film thickness and wavelength. Measurements in combination with an STM demonstrate a new distance dependence of the optical signal, which seems to reflect the phase sensitivity of the optical signal. We start with calculations based on the solution of Maxwell's equations for a layered system in a transfermatrix formalism [9] in order to motivate the background problem as well as the proposed solution. Then, we describe the experimental setup and results.

\section{Calculations}

In Fig. 1 the calculated surface-plasmon resonances are shown as function of the incident angle for the system glass (BK 7), $51 \mathrm{~nm} \mathrm{Ag}$, air for three different wavelengths with p-polarized light. Clearly, one can observe the problem already addressed in the introduction. The minimum value of the resonance, which defines the background intensity varies from $2.3 \%(760 \mathrm{~nm})$ to $10 \%(633 \mathrm{~nm})$ and $22 \%(514 \mathrm{~nm})$, respectively. In Fig. 2 the relative phase of the reflected light is shown. A phase change of $2 \pi$ is observed for $p$-polarized light from one side of the resonance to the other. The steepness of the transition is directly connected with the half-width of the resonance. Also shown in Figs. 1 and 2 is the behaviour of s-polarized light for one particular wavelength $(760 \mathrm{~nm})$. Independent of the wavelength, both the reflected intensity (Fig. 1) as well as the phase (Fig. 2) show only negligible variations. This has been already used in other experiments, where the s-polarized beam served as a reference to measure absolute phase shifts [10]. For the optimization of the background signal, which we address here, the s-polarized beam can be used in the following way: A mixed beam of s- and p-polarized light is produced by a $\lambda 2$ plate. The

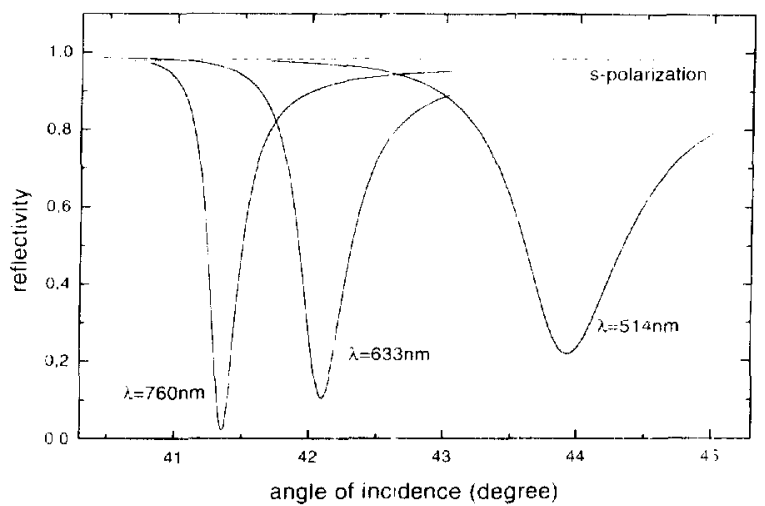

Fig. 1. Calculated reflectivity of the system glass. 51. $\mathrm{nm} \mathrm{Ag}$, air as function of incidence angle for p-polarization $(\lambda=760.633$. $514 \mathrm{~nm})$ and s-polarization $(\lambda=760 \mathrm{~nm}$, dashed line).

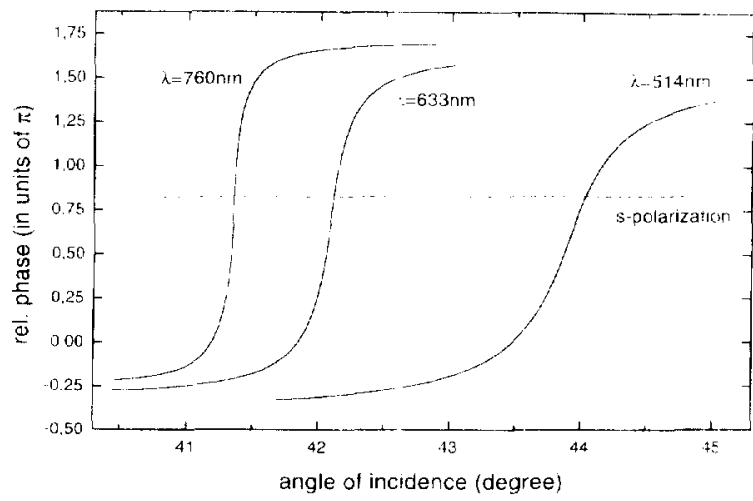

Fig. 2. Calculated phase of the system glass, $51 \mathrm{~nm} \mathrm{Ag,} \mathrm{an} \mathrm{dis}$ function of incidence angle for p-polarization $i d=760,6.33$, $514 \mathrm{~nm})$ and s-polarization $(i=760 \mathrm{~nm}$, dashed line).

intensity (and thus the electric field amplitude) of the s-polarized part is chosen equal to the minimum intensity of the resonance curve, which was obtained with p-polarized light. The relative phase between s- and p-polarized beam is adjusted with a Soleil-Babinet compensator to reach $\pi$ after the prism. Therefore we end with a reflected beam with same electric field amplitude in both polarizations and a phase shift of $\pi$ between s- and p-polarized part. This allows now to minimize the reflected intensity through destructive interference by a polarizer oriented 45 with respect to s-and p-polarized light. Furtheron, we call this prefered situation 


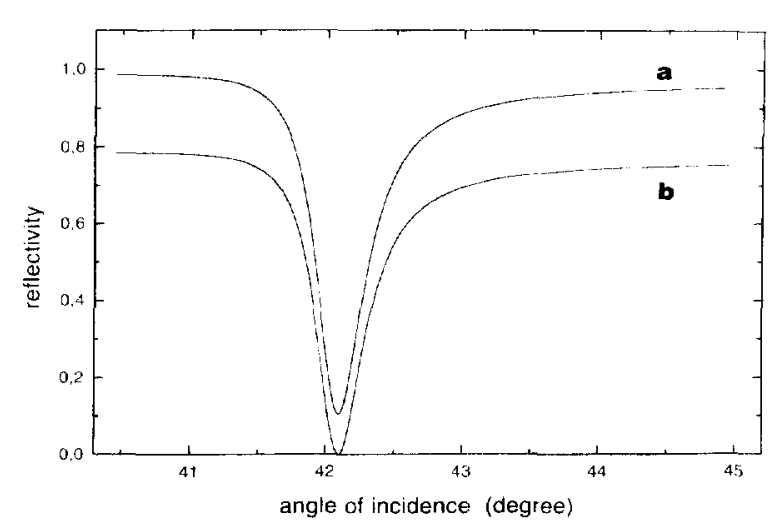

Fig. 3. Calculated reflectivity of the system glass, $51 \mathrm{~nm} \mathrm{Ag}$, air as function of incidence angle for pure p-polarized (a) and MR-light (b) $(\lambda=633 \mathrm{~nm})$.

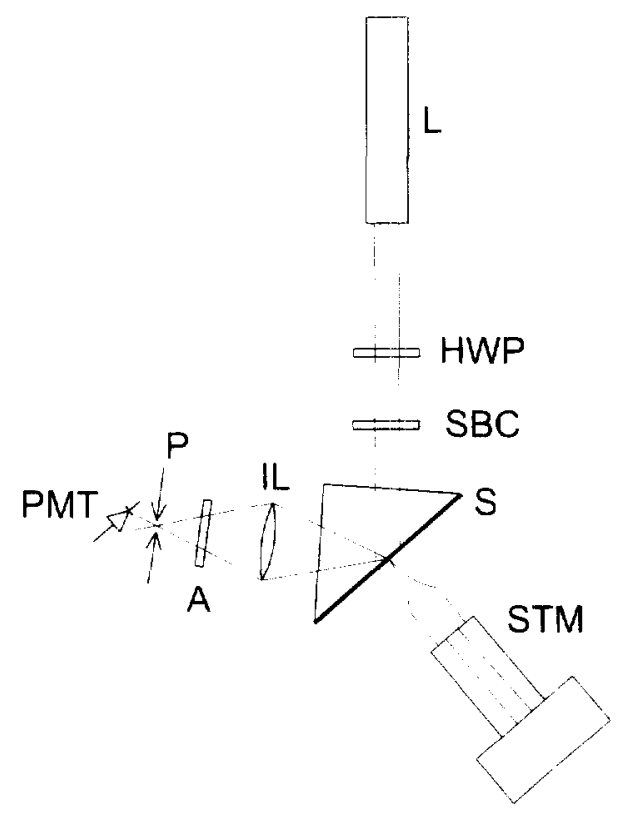

Fig. 4. Experimental setup consisting of laser (L). half-wave plate (HWP), Soleil-Babinet compensator (SBC), sample (S). imaging lens (IL), analyser (A), pinhole ( $P$ ), photomultiplier tube $(\mathrm{PMT})$ and scanning tunnelling microscope (STM).

of incoming electric field amplitude and phase, state of minimized reflectivity (MR).

Fig. 3 shows a comparison of the resonance behaviour with pure p-polarized and MR-light for $i=633 \mathrm{~nm}$. As expected the reflected intensity reaches zero in the resonance minimum. At the same time the addition of the s-polarized beam changes the intensity of the reflected beam outside the resonance minimum too. As both the phase change and the intensity of the p-polarized beam are strongly angle-dependent (Fig. 1a and Fig. 1bi the reflected intensity after the polarizer has to be calculated directly from the complex electric field components.

\section{Experimental setup}

A cw HeNe-laser ( $\lambda=633 \mathrm{~nm}, 6 \mathrm{~mW}$, polarization ratio $\left.<10^{-3}\right)$ and a $\mathrm{cw}$ Ti : sapphire $(\lambda=$ $760 \mathrm{~nm}, 100 \mathrm{~mW}$, polarization ratio $<10^{* 3}$, are used to excite surface plasmons in the Kretschmann attenuated total reflection geometry [11]. A half-wave plate and a Soleil-Babinet compensator allow to adjust the relative phase and amplitude of s- and p-polarized light (Fig. 4). The surface plasmons are excited at the surface of a silver film with thickness $51 \mathrm{~nm}$ which was evaporated on the base of a $\mathrm{BK} 7$ prism in a high vacuum chamber (pressure $5 \times 10^{-1 "}$ mbar during evaporation). The thickness of the evaporated film is controlled by a quartz microbalance.

The detection arm of the experiment consists of an objective which images the area of the silver surface onto the detector, where later the tip of the scanning tunnelling microscope approaches the surface. After the imaging objective the beam passes a polarizer, fixed at an angle of $45^{\circ}$ towards both s- and p-polarization. The transmitted intensity is measured with a photomultiplier. A $10 \mu \mathrm{m}$ pinhole in front of the photomultiplier is used for a further reduction of the detection area.

The home-made scanning tunnelling microscope is described in detail elsewhere [12]. For the experiment we used electrochemically etched PtIrtips [13].

\section{Experimental results and discussion}

The first experiments were performed to verify the calculated improvements in the signal back- 

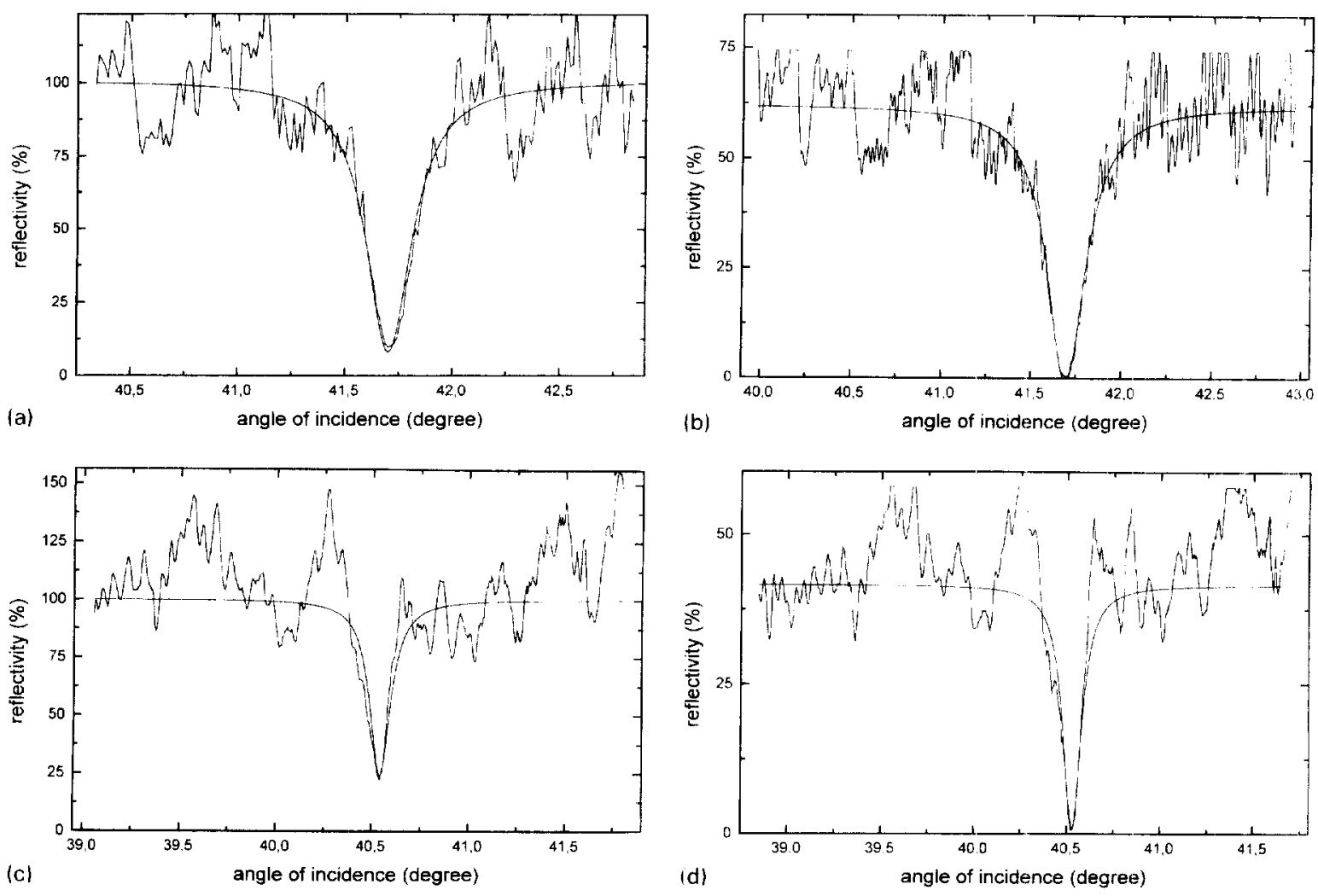

Fig. 5. Measured reflectivity as function of incidence angle; (a) p-polarized, $\lambda=633 \mathrm{~nm}$. (b) MR-light, $\lambda=633 \mathrm{~nm}$, (c) p-polarized, $\hat{\lambda}=760 \mathrm{~nm}$ and (d) MR-light, $\lambda=760 \mathrm{~nm}$. The experimental data were normalized on a value outside the resonance measured with pure p-polarized light and fitted to a Lorentzian curve for better visibility.

ground ratio. Therefore, the intensity at the detector was measured once with pure p-polarized and afterwards with MR-light. Fig. 5 shows the results of the measurements of the resonance curve for two wavelengths. The reflectance was normalized on the value outside the resonance measured with pure p-polarized light. The increased noise level outside the minimum is due to interferences in the optical path. In order to improve the visibility we added a Lorentzian fit to the data points. The position of the minimum is slightly shifted compared to the calculations. No effort has been made to reduce this discrepancy through an optimization of the optical constants used in the calculations. Nevertheless, the effect of the MRlight can clearly be observed in the measurements.
At $633 \mathrm{~nm}$ the minimum intensity with p-polarized light is $8 \%$. The MR-light reduces this intensity towards $0.25 \%$, which is a factor of around 30 . An even higher reduction (from $24 \%$ to $0.5 \%$ ) is found for $760 \mathrm{~nm}$. For the measurements with the STM the gain in the signal/background ratio can be estimated to be a little bit smaller than this factor. As a small amount of the incident laser intensity has to be transformed into s-polarized light, the field at the surface which defines the interaction with the tip of the STM is reduced as well.

In order to study the compatibility of the ellipsometric setup with the STM we approached the STM-tip to the surface. Fig. 6 shows the behaviour of the reflected intensity in the resonance minimum 


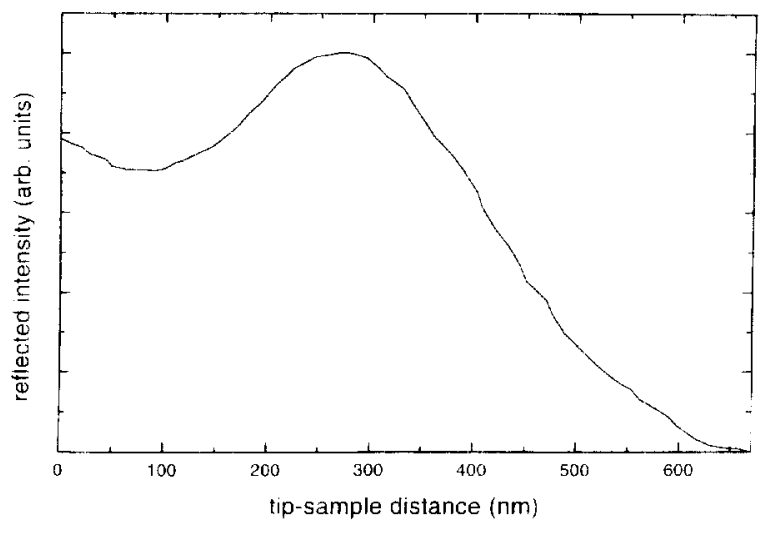

Fig. 6. Reflected intensity in the resonance minimum as function of tip-sample distance for $\lambda=633 \mathrm{~nm}$.

measured with MR-light for $i=633 \mathrm{~nm}$ as function of the tip-sample distance. The onset of tunneling was used to define the zero of the $x$-axis. From $700 \mathrm{~nm}$ to about $300 \mathrm{~nm}$ distance, an expected behaviour can be observed. The measured light intensity increases with approaching tip. This has been observed in other experiments as well $[3,5]$. On further approaching the surface the reflected intensity first decreases between 300 and $80 \mathrm{~nm}$ and then increases again. A similar dependence has not been observed in experiments without phase contrast [3,5].

This behaviour is not easily interpreted. One possible model would be the disturbance of the local surface-plasmon resonance conditions by the STM-tip. Then upon approaching the tip, the resonance angle should shift. As in our STM-experiments the measuring angle is fixed in the original minimum, an angular shift would mean an increase in reflectivity as well as a phase shift (Figs. 1 and 2). With further phase shift the destructive interference with the s-polarized light gets worse, so also the phase shift increases the measured reflectivity. Thus, this model cannot explain the decrease in reflectivity below $300 \mathrm{~nm}$ tip-sample distance.

In another feasible model, the tip acts as scattering centre for the local plasmon field. This has been used successfully to explain the distance dependence in similar experiments without phase contrast [3]. Once again the scattered intensity should in- crease upon approaching the tip, as the plasmon field decays exponentially above the silver surface. In order to explain the measured distance behaviour, a distance-dependent phase shift of the scattered light has to be assumed. Up to now this is a speculation, but the situation can be clarified by a whole set of experiments with slightly changed angle of incidence and variable phase shift.

In conclusion, our experiments have shown that the addition of an ellipsometric setup to a SPNM allows to improve the signal/background ratio considerably. This method allows to make spectroscopic measurements feasible without changing the metal film thickness. Furthermore, it may be possible to achieve phase information with this setup in an easy manner, as the reference beam is automatically included in the experiment.

\section{Acknowledgements}

We thank S. Herminghaus and C. Bechinger for stimulating discussions and the $\mathrm{DFG}$ (contract number BO1079/3) and the "Zentrum für moderne Optik Konstanz" for financial support.

\section{References}

[1] F. Zenhausen. M.P. O'Boyle, H.K. Wickramasinghe, Appl Phys. Lett. 65 (1994) 1623.

[2] Y. Martin, F. Zenhausen, H.K. Wickramasinghe, Appl Phys. Lett. 68 (1996) 2475

[3] M. Specht, J.D. Pedarnig, W.M. Heckl, T.W. Hänsch. Phys. Rev. Lett. 68 (1992) 476

[4] J.D. Pedarnig, M. Specht, W.M. Heckl, T.W. Hänsch Appl. Phys. A 55 (1992) 476.

[5] R.B.G. de Hollander. N.F, van Hulst, R.P.H. Kooymann. Ultramicroscopy 57 (1995) 26 ?

[6] J. Koglin, U.C. Fischer, H. Fuchs, I. Biomedical Opt. $1(1996) 75$.

[7] Y.-K. Kim, P.M. Lundquist, I.A. Helfrich, J.M. Mikrut. G.K. Wong, P.R. Auvil, J.B. Ketterson, Appl. Phys. Lett. 66 (1995) 3407.

[8] H. Raether, Surface Plasmons, Springer Tracts in Modern Physics. vol. 111, 1988. 
[9] R.M.A. Azzam, N.M. Bashara, Ellipsometry and Polarized Light, North-Holland, Amsterdam, 1987.

[10] S. Herminghaus, C. Bechinger, W. Petersen, P. Leiderer, Opt. Commn. 112 (1994) 16.
[11] E. Kretschmann. Z. Fhys. 241 (1971) 313.

[12] F. Mugele, Ch. Kloos, P. Leiderer, R. Möller, Rer. Sci Instrum. 67 (1996) 2557

[13] A.J. Melmed, J. Vac. Sci. Technol. B $9(1991) 601$ 